\title{
Research on the Optimization of the Transportation of the Hazardous Materials
}

\author{
Guo Fengrun ${ }^{1, a}$, Liu Weidong ${ }^{2, b}$, Han Wentao ${ }^{2, c}$ and Wei $\mathrm{Yu}^{1, d}$ \\ ${ }^{1}$ Post-graduate Department, Engineering University of CAPF, Xi'an 710086, China \\ ${ }^{2}$ Equipment and Transportation Department, Engineering University of CAPF, Xi'an 710086, China \\ a467590570@qq.com, bguofengrun@126.com, hanwentao_1@sohu.com, 467539628@qq.com
}

Keywords: Hazardous materials; Transportation;Ways;Optimization

Abstract: Many ways may be available to transport the hazardous materials. However, different ways will lead to the difference in the cost of transportation and loss of total social expectations. Under the same condition, this paper studies the best way and path to move the materials of danger. It explains the solving method of the model by a simulation example. The comparison of the various ways of transportation shows that a combination of different ways can lower the cost of the transportation as well as the loss of the total social expectations.

\section{Introduction}

With the rapid growth of the economy, an increasing number of the hazardous materials need to be transported, adding more risks to the road safety. The probability of an accident and the cost vary from ways of transportation to the paths that are chosen. Many scholars at home and abroad have done some researches on how to choose the best way and path to transport the materials in order to lower the cost of transportation and the loss of any possible accidents. The Literature [1] analyzes how the government reduces the risk of transporting the hazardous materials in the existing networks by closing networks of some road sections without undermining the economic interests of transportation. The primary model is to provide the certain path for every carriage, which means that under the condition of reducing the risks, the cost of transportation will be taken into consideration and the path of no economic interests will be ruled out.

Given that the government offers different loss of total social expectations and cost of transportation in accordance with the different kinds of hazardous materials in the exiting transportation networks. Literature[3] combines some ways of transporting and increases possible cost of transportation and the fees for transfer while transferring materials in the transit point, which will make in more real. This paper takes hazardous materials, ways and paths of transportation into account and finds the best way and path for transporting materials in the condition of lowest loss of the accidents and cost of the transportation by making a comparison between using the single way and combining several ways.

\section{The components of the hazardous materials transporting system}

The hazardous materials transporting system consists of people(driver, pedestrian, residents), mechanicals(transportation vehicles, facilities), hazardous materials and condition(weather, road, geography)and so on. The factors affect each other. Once there is something wrong with one of them, an accident will happen, causing personal injury and loss of property. Therefore, it is necessary to keep a coordinated and harmonious operation among people, mechanicals and condition, which also serves the basic principles of the coordination among those three factors.

The model of optimizing the ways of transporting hazardous materials and the path of transportation 
The paper describes its studying issues as follows: In the transporting network with many nodes, there exists various ways of transportation. Here is an assumption that some hazardous materials need to be transported and one can only choose one way between the two nodes. The data are known including the costs for transporting and transferring at the transmit point as well as the probability of an accident and its loss. One needs to determine the best way and path of transportation, considering such conditions as the cost of transportation and the loss of an accident.

If $\mathrm{G}=(\mathrm{N}, \mathrm{E})$ represents a transportation network of one hazardous materials and $\mathrm{N}=$ node set ( Starting, final and transit point ), E=edge set and $l$ represents certain liquidity of hazardous materials in the G network and the starting point is $s(l)$, final point is $f(l)$, a set of the total liquidity is L, then $l \in L ; h$ is one way of transportation in the G network and only one way can be chosen between two nodes and $H$ is the set of all ways, then $h \in H ; n^{l}$ means transit node of hazardous materials of the $l$ liquidity in the network $\left(n^{l} \neq \mathrm{s}(l), n^{l} \neq f(l)\right.$ ), the set of $n^{l}$ is $N^{l}, W_{k}^{l}$ means the optional ways of the hazardous materials of the $l$ liquidity in the G network. The set of the optional ways is $W^{l}$,then $W_{k}^{l} \in W^{l}$;If only one kind of the hazardous materials in the network needs to be transported, $m^{l}$ is the transportation volume of the $l$ liquidity, then the $P_{i j}^{h}$ is the probability of the accidents happening in the road section $(i, j)$ of the transportation way $h, P^{h_{x} h_{y}}$ is the probability of the accidents happening in transferring the materials in the transit of the transportation way $h_{x}$ and $h_{y}, c_{i j}^{h}$ represents the costs of transporting per unit hazardous materials in the road section $(i, j)$ by the transportation way $h ; c^{h_{x} h_{y}}$ represents the costs of transferring per unit hazardous materials in the transit by the transportation way $h_{x}$ and $h_{y} ; r_{i j}^{h}$ represents the loss of the accident happening while transporting per unit hazardous materials in the road section $(i, j)$ by the transportation way $h ; r^{h_{x} h_{y}}$ is the loss of the accident happening while transferring per unit hazardous materials in the transit by the transportation way $h_{x}$ and $h_{y}$;

Defining the decision variables as follows:

$$
=\left\{\begin{array}{l}
x_{i j}^{l h} 1, \text { transportation way } h \text { chosen by the } l \text { liquidity in the } \operatorname{road} \operatorname{section}(i, j) \\
0, \text { others }
\end{array}\right.
$$

The total cost in the transportation network is composed by the transportation cost in the road section and the cost in the transit node, which can be shown as follows:

$$
\begin{aligned}
& w_{1}=\sum_{l \in \mathrm{L}} \sum_{\mathrm{W}_{\mathrm{k}}^{1} \in W^{l}} \sum_{h \in H} \sum^{(i, j) \in W_{k}^{l}} x_{i j}^{l h} m^{l} r_{i j}^{h} P_{i j}^{h} \\
& +\sum_{\mathrm{l} \in \mathrm{L}} \sum_{\mathrm{W}_{\mathrm{k}}^{1} \in W^{l}} \sum^{h_{x} \in H} \sum^{h_{y} \in H} \sum^{\left(i, n^{l}\right) \in \mathrm{W}_{\mathrm{k}}^{1}} \sum^{l} \sum^{l, j) \in \mathrm{W}_{\mathrm{k}}^{1}} x_{i n^{l}}^{l h_{x}} x_{n^{l} j}^{l h_{y}} m^{l} P^{h_{x} h_{y}} r^{h_{x} h_{y}}
\end{aligned}
$$

The loss causing by the accident in the transportation network consists of the loss causing by the accident in the road section and the loss causing by the accident in the transit node, which is shown as follows:

$$
\begin{aligned}
& w_{2}=\sum_{\mathrm{l} \in \mathrm{L}} \sum_{\mathrm{W}_{\mathrm{k}}^{l} \in W^{l}} \sum_{h \in H} \sum^{(i, j) \in W_{k}^{l}} x_{i j}^{l h} m^{l} c_{i j}^{h} \\
& +\sum_{\mathrm{l} \in \mathrm{L}} \sum_{\mathrm{W}_{\mathrm{k}}^{1} \in W^{l}} \sum^{h_{x} \in H} \sum^{h_{\mathrm{y}} \in H} \sum^{\left(i, n^{l}\right) \in \mathrm{W}_{\mathrm{k}}^{1}} \sum^{\left(n^{l}, j\right) \in \mathrm{W}_{\mathrm{k}}^{1}} x_{i{ }^{l}}^{l h_{x}} x_{n^{l} j}^{l h_{y}} m^{l} c^{h_{x} h_{y}} \\
& \text { Published by Atlantis Press, Paris, France. } \\
& \text { (C) the authors }
\end{aligned}
$$


Weighting two target functions in a linear method and giving the respective weightiness of the loss of the total social expectation and the cost of the transportation (Weighting coefficient $\alpha$ meets the condition $0 \leq \alpha \leq 1$ ), the target function of the problem is:

The constraint condition of the model is:

$$
\begin{aligned}
& W=\min \left\{\alpha w_{1}+(1-\alpha) w_{2}\right\} \\
& \sum_{(i, j) \in W_{k}^{l}} x_{i j}^{l h}-\sum_{(j, i) \in W_{k}^{l}} x_{j i}^{l h}= \\
& \left\{\begin{array}{rl}
1 & \mathrm{i}=\mathrm{s}(l) \\
-1 & \mathrm{i}=\mathrm{f}(l), l \in \mathrm{L}, W_{\mathrm{k}}^{l} \in W^{l}
\end{array}\right. \\
& \sum^{h \in H} x_{i j}^{l h} \leq 1, \quad l \in \mathrm{L} \\
& x_{\mathrm{ij}}^{l h} \in\{0,1\} \\
& W^{l} \geq 0, \quad l \in L
\end{aligned}
$$

The functions of all the constraint conditions are as follows:

$$
\left\{\begin{array}{l}
\text { constraint condition(1): } \text { to ensure the direction of the traffic flow and flow balance } \\
\text { constraint condition(2): } \text { to ensure only one transportation way available in each road section } \\
\text { constraint condition(3): to keep decision variables } 0 \text { or } 1 \\
\text { constraint condition(4): } \text { to keep the transportation volume of the various flows } \\
\text { non-negative. }
\end{array}\right.
$$

\section{Analysis of examples}

If there is a network diagram of the transportation of the hazardous materials as Diagram 1 and it has six nodes and eight road sections, then $\mathrm{N}=\{1,2,3,4,5,6\}, \mathrm{E}=\{(1,2),(1,3),(2,4),(2,5),(3,5),(4$, $6),(5,4),(5,6)\}$. Two ways of transportation in this network are $h_{x}$ and $h_{y}$ in which $2 \rightarrow 4$ can only use transportation way $h_{x}$ and $5 \rightarrow 4$ can use the way $h_{y}$.If one kind of hazardous materials of liquidity $f$ needs to be transported from starting point 1 to the final point 6 , then the transportation volume is $m^{l}=50$

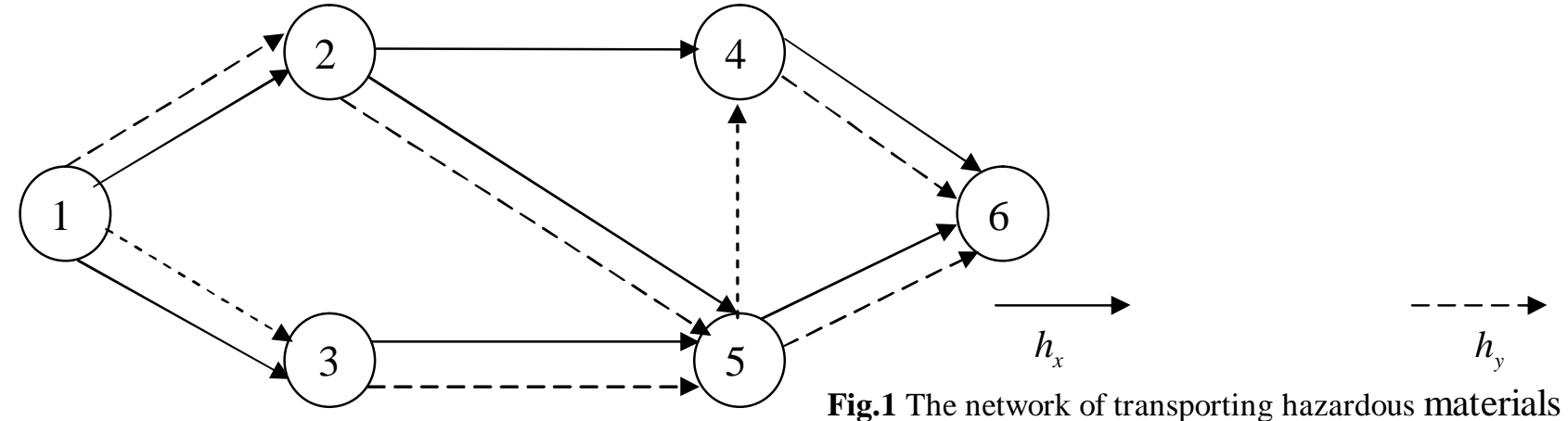


The transferring node of the liquidity $f$ from Diagram 1 is $\mathrm{N}=\{2,3,4,5\}$.The set of the paths is $E=\left\{E_{1}, E_{2}, E_{3}, E_{4}, E_{5}\right\}$, then $E_{1}: 1 \rightarrow 2 \rightarrow 4 \rightarrow 6 ; E_{2}: 1 \rightarrow 2 \rightarrow 5 \rightarrow 4 \rightarrow 6 ; E_{3}: 1 \rightarrow 2 \rightarrow 5 \rightarrow 6 ; E_{4}: 1 \rightarrow 3 \rightarrow 5 \rightarrow$ $4 \rightarrow 6 ; E_{5}: 1 \rightarrow 3 \rightarrow 5 \rightarrow 6$.

The probability of accidents happening in every road section in the transportation ways $h_{x}$ and $h_{y}$ is respectively $P_{i j}^{h_{x}}$ and $P_{i j}^{h_{y}}$. The loss of per unit hazardous materials after the accidents is $r_{i j}^{h_{x}}$ and $r_{i j}^{h_{y}}$ and the costs of transportation is $c_{i j}^{h_{x}}$ and $c_{i j}^{h_{y}}$ as they are shown in Table 1.

The probability of accidents happening in transit point in the transportation ways is $P^{h_{x} h_{y}}$ and the loss of per unit hazardous materials after accidents is $r^{h_{x} h_{y}}$ and the cost of transferring is $c^{h_{x} h_{y}}$ as they are shown in the Table 2.

Table 1 The probability of the two transportation ways in everyroad section, the loss of per unit hazardous materials after accidentsand the cost of the transportation

\begin{tabular}{|l|c|c|c|c|}
\hline & $1 \rightarrow 2$ & $1 \rightarrow 3$ & $2 \rightarrow 4$ & $2 \rightarrow 5$ \\
\hline$h_{x}$ & $0.01 / 2200 / 34$ & $0.02 / 1800 / 40$ & $0.02 / 1400 / 50$ & $0.01 / 3800 / 40$ \\
\hline$h_{y}$ & $0.02 / 7000 / 20$ & $0.008 / 1250 / 16$ & - & $0.03 / 1000 / 32$ \\
\hline & $3 \rightarrow 5$ & $4 \rightarrow 6$ & $5 \rightarrow 4$ & $5 \rightarrow 6$ \\
\hline$h_{x}$ & $0.01 / 1800 / 20$ & $0.02 / 1100 / 38$ & - & $0.04 / 550 / 24$ \\
\hline$h_{y}$ & $0.05 / 920 / 60$ & $0.01 / 3000 / 50$ & $0.02 / 500 / 14$ & $0.01 / 2800 / 32$ \\
\hline
\end{tabular}

Table 2 The probability of the two transportation waysin the transit point, the loss of per unit hazardous materialsafter accidents and the cost of the transferring

\begin{tabular}{|l|c|c|}
\hline & $h_{x}$ & $h_{y}$ \\
\hline$h_{x}$ & - & $0.005 / 2000 / 20$ \\
\hline$h_{y}$ & $0.01 / 1600 / 18$ & - \\
\hline
\end{tabular}

If $\alpha=0.4$, putting the relevant data into the model and using the Lingo90, the optimal value is6278, then $x_{13}^{l h_{x}}=1, x_{35}^{l h_{y}}=1, x_{56}^{l h_{y}}=1$, the results of the transportation ways and the optimization of the transportation paths are $: 1 \rightarrow h_{y} \rightarrow 3 \rightarrow h_{x} \rightarrow 5 \rightarrow h_{x} \rightarrow 6$, the loss of total social expectation is 3300,the cost of the transportation is 3900 .

If $\alpha=0.9$, putting the relevant data into the model and using the Lingo90, the optimal value is 5000, then $x_{12}^{l h_{x}}=1, x_{24}^{l h_{y}}=1, x_{46}^{l h_{y}}=1$, the results of the transportation ways and the optimization of the transportation paths are $: 1 \rightarrow h_{x} \rightarrow 2 \rightarrow h_{x} \rightarrow 4 \rightarrow h_{x} \rightarrow 6$, the loss of total social expectation is 2600 , the cost of the transportation is 4600 .

The different values show that the best way and path for transporting hazardous materials is also different. Sometimes it can be a single way and sometimes it can be a combination of several ways. When $\alpha=0.9$, it is a single way and when $\alpha=0.4$, it is a combination of two ways. Taking $\alpha=0.4$ as an example, if the way of $1 \rightarrow h_{y} \rightarrow 3 \rightarrow h_{y} \rightarrow 5 \rightarrow h_{y} \rightarrow 6$ is taken, then the loss of the total social expectation and the cost of transportation are 84 and 108 respectively. If materials is transferred in the other way at the node 3 , which is the way of $1 \rightarrow h_{y} \rightarrow 3 \rightarrow h_{x} \rightarrow 5 \rightarrow h_{x} \rightarrow 6$, then the loss of the total social expectation of the per unit of hazardous materials and the cost of transportation are 66 and 78 . Therefore, in the proper condition, combining several ways of transportation can lower the loss of the total social loss and the cost of the transportation. 


\section{Conclusion}

In the real transportation, there may be many ways of transportation such as by land, train, water and air in each node. According to the different hazardous materials, one can choose the single way or the combination of some ways. By considering the cost in transporting the hazardous materials and the loss of total social expectation, we will regard the minimum weighted linear of the cost of the transportation and the loss of the total social expectation as the target function and take different weight coefficients in line with the corresponding kinds of hazardous materials. The model building on that is of certain generality is able to determine the best way to transport different hazardous materials and lower the transportation cost of per unit hazardous materials and the loss of total social expectation to some extent.

\section{References}

[1] Vedat V,Bahar YK. A Path. Based Approach for Hazmat Transport Network Design[J] . Management Science,2008,1(54):29 40.

[2] Erkut E,Gzara F. Solving the hazmat transport network design problem [J]. Computers \& Operations Research,2008, 35( 7): 2234 2247

[3] Kai Yanxia,Wang Haiyan. Research on optimization of transportation mode and route for hazardous materials transportation network $[\mathrm{J}]$. Journal of Safety Science and Technology. 2009,(5):37 41

[4] Zhou Furong, Gao Huiying,Su Na. On the policy of the transportation paths of hazardous materials in urban $\operatorname{road}[\mathrm{J}]$. Journal of management science

[5] Shi Lichen,Wei Lijun,Wu Zongzhi. On the Optimization of multi-target path of transporting hazardous materials in the road[J] .Journal of Safety Science and Technology. 2006,5(2):59 63

[6] Ren Changxing,Wu Zongzhi. Probe into Methods of Optimal Road Transportation Routing for Hazardous Materials [J] . China Safety Science Journal. 2006,16(6):129 134

[7] Wang Haiyan,Wang Hongguang. Designing Emergency Response Networks for Hazardous Materials Logistics Based on Maximal Cover of Population Risk,Proceedings of the 2008 International Conference On e-Risk Management(ICeRM 2008), Atlantis Press,2008,582 589

[8] Shorys D. A Model for the selection of shipping routes and storage location for a hazardous substance[D] . Johns Hopkins University. Baltimore,1981

[9] ZHANG De-zhi,LING Chun-yu. A combination optimization model for multiple transportation mode selection and solution algorithm[J].Journal of Railway Science and Engineering,2002,4(20) . $71 \sim 75$

[10] Erkut E, Gzara F. Solving the hazmat transport network design problem[ J ]. Computers \& Operations Research, 2008. 35: 2234 2247.

[11] R.Bubbico. Maschio,B.Mazzarotta. Risk management of road and rail transport of hazardous materials in Sicily[J] . Journal of Loss Prevention in the Process Industries,2006, (1 9):32 38 